\title{
Antiinflamatórios Não Hormonais Na Clínica Médica-Odontológica
} Non-Steroidal Anti-inflammatory Drugs in the Medical and Dental Clinics

\author{
ROSIMAR DE CASTRO BARRETO' \\ MARGARETH DE FÁTIMAF. MELO DINIZ ${ }^{2}$ \\ GIUSEPPE ANACLETO SCARANO PEREIRA ${ }^{3}$ \\ HELLEN ROSI BARRETO CELANI ${ }^{4}$
}

\section{RESUMO}

Objetivo: Este estudo teve como objetivo revisar a literatura científica sobre o uso racional dos anti-inflamatórios não esteroides na clínica médica-odontológica, tendo como primeira opção a sua escolha nos mais variados processos inflamatórios que requeiram a sua administração e que em contrapartida venham a proporcionar menos efeitos adversos sobre o paciente. Material e métodos: Foi consultada a rede BVS de dados tendo como o descritor os Anti-inflamatórios não esteroides. Resultados: A literatura relata que ao inibirem a síntese de prostaglandinas e tromboxano, os anti-inflamatórios não hormonais (AINEs), são os mais utilizados no controle das manifestações musculoesqueléticas, sendo amplamente utilizados na Clínica Médica e Odontológica para prevenir as reações inflamatórias traumáticas pós-cirúrgicas. Todavia estes medicamentos podem provocar uma série de efeitos colaterais quando mal empregados. $O$ processo doloroso que acomete as estruturas bucais, geralmente é de origem inflamatória aguda, obrigando o paciente a buscar um cirurgião-dentista para tratá-las adequadamente, no entanto o profissional deve ter um bom conhecimento sobre as suas indicações, contra-indicações e efeitos adversos. São prescritos preferencialmente os inibidores seletivos da COX-2, sendo portanto os mais indicados, por apresentarem melhor eficácia anti-inflamatória e sem os efeitos gastrintestinais indesejáveis são chamados de coxibes, se ligam seletivamente ao local ativo da enzima COX-2 e a bloqueia com mais eficácia que a COX-1. Conclusão: Nesta revisão pode ser aferido que os COX-2, além de serem os mais indicados, têm também efeito analgésico e anti-inflamatório semelhante aos demais AINE'S, estando portanto indicado como sendo de primeira escolha para o tratamento de pacientes que têm predisposição a ulceração e sangramento digestivo.

\section{DESCRITORES}

Anti-inflamatórios não-esteroides. Ciclooxigenase. Inflamação.

\begin{abstract}
Objective: This study aimed to review the scientific literature on the rational use of non-steroidal anti-inflammatory drugs in the medical and dental clinics, based on the treatment of inflammatory processes with fewer adverse effects. Material and Methods: Bibliographical searches were carried out in the electronic database BVS using the descriptor "non-steroidal anti-inflammatory". Results: The literature reports that by inhibiting the synthesis of prostaglandins and thromboxane, non-steroidal anti-inflammatory drugs (NSAIDs) have been mostly used in medicine and dentistry for the control of musculoskeletal manifestations to prevent post-surgical traumatic inflammatory reactions. However, these drugs can cause a number of side effects when misused. The painful process that affects the oral structures is usually of acute inflammatory origin, forcing the patient to seek a dentist to properly treat them. Hence, the professional should have a good knowledge of the drug indications, contraindications and side effects. Selective inhibitors of COX-2 are being preferably prescribed as these have better anti-inflammatory effectiveness and no undesirable gastrointestinal effects. These drugs are called coxibs and selectively bind to the active site of the enzyme COX-2, creating a blockage which is more effective than that of COX-1. Conclusion: This review may infer that COX-2 NSADs are most commonly indicated due to their analgesic and anti-inflammatory effectiveness, which is similar to that of other NSAIDs. Thus, this class of drugs is indicated as a first choice for the treatment of patients who are predisposed to ulceration and digestive bleeding.
\end{abstract}

\section{DESCRIPTORS}

Anti -inflammatory non-steroidal. Cyclooxygenase . Inflammation.

\footnotetext{
Professor Titular do Departamento de Clínica e Odontologia Social da Universidade Federal da Paraíba (DCOS/UFPB), João Pessoa/PB, Brasil. Professora Titular do Departamento de Ciências Farmacêuticas da Universidade Federal da Paraíba (DCF/UFPB), João Pessoa/PB, Brasil.

3 Professor Associado do Departamento de Clínica e Odontologia Social da Universidade Federal da Paraíba (DCOS/UFPB), João Pessoa/PB, Brasil

4 Cirurgiã-Dentista Especialista em Odontologia Hospitalar, Hospital Santa Isabel, João Pessoa/PB, Brasil.
} 
$\mathrm{O}$ anti-inflamatórios não hormonais (AINE's) do nglês, Non-steroidal anti-inflammatory drug, representam uma das classes de medicamentos de maior aplicação dentro da clínica médicaodontológica. São constituídos por grupos químicos heterogêneos de fármacos que têm em comum a capacidade de controlar a inflamação, produzir analgesia e combater a febre ${ }^{1,2}$. Na sua essência tem como ação impedir a síntese de eicosanoides pela via metabólica da cascata do ácido araquidónico4, e são inibidores específicos da enzima ciclo-oxigenase (COX), existindo dois tipos e formas diferentes, designadas COX-1 e COX2. A descoberta dos dois subtipos de COX criou expectativas quanto à criação de novos fármacos que mantivessem as mesmas propriedades dos AINE's existentes e que viessem permitir a diminuição da incidência de efeitos colaterais ${ }^{3}$. Os diferentes subtipos de COX levaram a que fosse colocada a hipótese de que a COX-1 seria "constitutiva", ou seja, estaria sempre presente no corpo e era responsável por funções fisiológicas importantes, e que a COX-2 seria "induzida", surgindo na resposta inflamatória. Desta forma, os efeitos colaterais dos AINEs dever-se-iam à inibição da COX-1 $1^{4,5,6}$.

É importante frisar que a COX-1 e não a COX2 é expressa como isoforma constitutiva, dominante nas células epiteliais gástricas, e constitui a maior fonte da formação de prostaglandinas citoprotetoras. A inibição da COX-1 nesse local é tida como, em grande parte, a responsável pelos eventos gástricos adversos que complicam o tratamento com os AINEs, fornecendo assim a razão para o desenvolvimento de AINEs específicos para inibição da COX-2 $2^{7,10}$. Por outro lado, a COX-2 é uma enzima induzida na inflamação, influenciando os eventos vasculares.

$\mathrm{Na}$ coagulação sanguínea todos os AINE'S são contraindicados, pois atuam diminuindo a agregação plaquetária. O AAS é a substância que mais inibe a função plaquetária. Esse efeito colateral é frequentemente benéfico em doentes com risco de infarto e acidente vascular encefálico ${ }^{8}$. Todavia essa inibição das plaquetas e da coagulação é contraindicada em pacientes que irão se submeter a processos cirúrgicos, o que é sempre aconselhável suspender o uso do antiinflamatório sete dias antes de qualquer intervenção ${ }^{4,8}$.

\section{REVISÃODALITERATURA}

O controle da dor e febre, associada ou não à inflamação, tem sido uma preocupação desde os primórdios da civilização. Da casca do salgueiro (Salix alba vulgaris), Leroux em 1827, isolou a salicina. Mais tarde, Piria, em 1838, isolou o ácido salicílico. Em 1844, Cahours isolou o ácido salicílico do óleo de gaultéria. Em 1860, Kolbe e Leutemann conseguiram obter o ácido salicílico através de síntese. E finalmente em 1899, foi introduzido na clínica o ácido acetilsalicílico ${ }^{3,11}$.

Devido à toxicidade, principalmente irritação gastrintestinal, procuraram sintetizar substâncias com menos efeitos adversos. Então, em 1950, desenvolveram a fenilbutazona, o primeiro anti-inflamatório nãosalicilato. Entretanto, foram observados efeitos adversos, relacionados à discrasia sanguínea, assim como agranulocitose, o que ocasionou o seu abandono. A partir de 1960, foram desenvolvidos outros derivados acídicos com ações analgésicas e anti-inflamatórias com maior eficácia e dotados de menos efeitos indesejáveis ${ }^{12,13}$.

Com a introdução dos inibidores seletivos da COX-2 na clínica, visando manter a eficácia antiinflamatória e desprovidos dos efeitos gastrintestinais indesejáveis, atualmente são disponibilizadosos chamados inibidores seletivos da COX-2, chamados de coxibes, que se ligam seletivamente ao local ativo da enzima COX-2 e a bloqueia com mais eficácia que a COX1. Nota-se que essa subclasse tem efeito analgésico e anti-inflamatório semelhante aos demais AINE's, estando, portanto, indicado como primeira escolha para o tratamento de idosos e pacientes predispostos à ulceração e sangramento digestivo ${ }^{14}$.

\section{Mecanismo de Ação}

Os AINE's são inibidores específicos da enzima cicloxigenase (COX). A COX possui duas formas diferentemente ativas, designadas COX-1 e COX-2. Estas desempenham importante papel na cascata do ácido araquidónico, pois transformam o mesmo pela ação da fosfolipase A2, em dois tipos de compostos, as prostaglandinas e os tromboxanos ${ }^{5}$, que são mediadores na inflamação e na dor, assim como em vários outros processos fisiológicos como na coagulação ${ }^{4}$ (fig.1). Com o aparecimento de fármacos que inibem especificamente a COX-2, designados por coxibs o primeiro elemento deste grupo foi o Rofecoxib (VIOXX), onde foi possível constatar uma quase completa redução dos efeitos colaterais ao nível gastrointestinal. Contudo, alguns estudos vieram colocar em causa o fundamento desta abordagem, ao demonstrar que a COX-2 também desempenhava um papel fisiológico protetor importante simultâneo tanto no estômago como no rim ${ }^{7,8}$.

A inibição destas enzimas pelos AINE's na redução da febre ou efeito antipirético é causada pela inibição da formação de prostaglandina E2 pela COX-1. 
Esta prostaglandina é um mediador importante para a ativação do centro nervoso no hipotálamo, regulador da temperatura corporal. O efeito analgésico é devido à inibição da produção local de prostaglandinas quando da inflamação. Estas prostaglandinas, se forem produzidas, vão sensibilizar as terminações nervosas locais, que será iniciada por outros mediadores inflamatórios como a bradicinina causando a dor.

\section{Classificação Química}

Os anti-inflamatórios não hormonais (AINE’s) são de natureza química muito variada, porém todos atuam praticamente pelo mesmo mecanismo de ação, inibindo a síntese do ácido araquidônico, impedindo a formação de prostaglandinas e outros mediadores químicos do processo inflamatório ${ }^{5,18}$.

\section{Indicações Clínicas}

Ao inibirem a síntese de prostaglandinas e tromboxano, os AINEs são úteis no controle das manifestações sintomáticas musculares e esqueléticas nos pacientes acometidos com artrite reumatóide, polimiosite, lúpus eritematoso sistêmico, esclerose sistêmica progressiva, poliartrite nodosa e espondilite anquilosante e traumas pós cirúrgico. Demonstram eficácia na dismenorreia primária, mastocitose sistêmica, serosites lúpicas, pleurite e pericardite. São também utilizados como adjuvantes no tratamento da gota aguda e em osteoartrose, artroplastia e fibrose cística ${ }^{11,14}$. No tratamento específico da gota aguda, é relatada a eficácia de alguns AINEs, tais como, etoricoxibe, celecoxibe, indometacina, naproxeno e o sulindaco ${ }^{11,12,15}$.

As indicações mais comuns de AINE's na infância e adolescência são no controle da febre, dores agudas e crônicas. O ácido acetilsalicílico, naproxeno, ibuprofeno e o tolmentin são os mais indicados na faixa etária pediátrica. Os inibidores seletivos da COX-2 são indicados nos pacientes que apresentam efeitos colaterais como no caso da intolerância gástrica não controlada pelos medicamentos gastroprotetores ${ }^{16,17,18}$. $\mathrm{Na}$ clínica os AINE's são indicados por vários especialistas, como pediatras, otorrinolaringologistas, reumatologistas, ginecologistas, ortopedistas e os cirurgiões bucomaxilo que na clínica odontologica são os maiores prescritores desses medicamentos.

\section{DISCUSSÃO}

Os anti-inflamatórios AINE's, atuam na biossíntese das prostaglandinas por inibição das COX, existindo em algumas situações especificidade de bloqueio de isoformas, determinando as subclasses existentes que são os inibidores não seletivos de COX-1 e inibidores seletivos da COX-2 $2,5,16$. As ações anti-inflamatória, antipirética e analgésica são também encontradas nos diversos AINEs, com exceção do paracetamol. De uma maneira em geral as reações decorrentes da utilização desses fármacos ocorrem de forma primária na produção diminuída de PG, prostanoide encontrado no organismo, tanto em processos fisiológicos como patológicos.

Tem sua principal indicação nas doenças

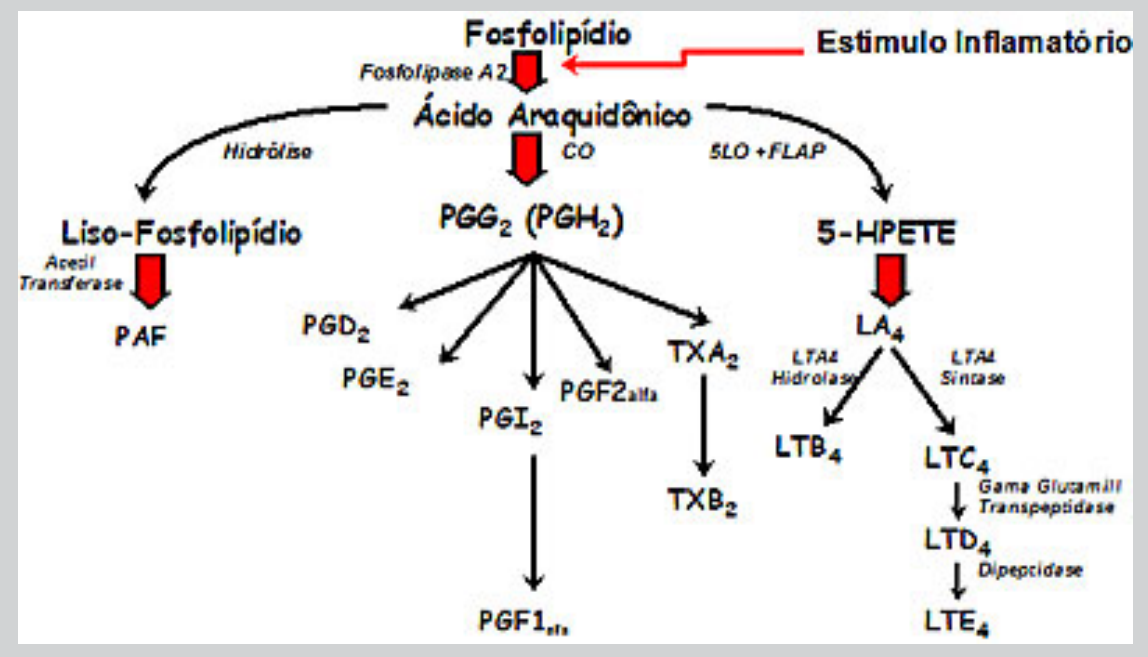

Figura 1. Síntese de eicosanoides cascata do ácido araquidônico

Fonte.http://www.asmabronquica.com.br/medical/mediadores_lipidicos.html 
Tabela 1. Classificacão dos Anti-inflamatórios não Esteróides (AINE's).

\section{Inibidores Não-Seletivos Da Cox}

Derivados do Ácido Salicílico (salicilatos)

Ácido Acetilsalicílico (Aspirina), Salicilato de Sódio, Salicilato de Metila, Diflunisal, Flunfenisal, Sulfassalazina, Olsalazina

Derivados Pirazolônicos

Dipirona. Fenilbutazona, Apazona, Sulfimpirazona

Derivados do Para-aminofenol

Paracetamol (Acetaminofeno)

Derivados do Ácido Indolacético e Ácido Indenoacético Indometacina, Sulindaco, Etodolaco

Derivados do Ácido N-fenilantranílico (fenamatos)

Ácido Mefenâmico, Ácido Meclofenâmico, Ácido Flufenâmico, Ácido Tolfenâmico, Ácido Etofenâmico

Derivados do Ácido Pirrolalcanoico

Tolmetino, Cetorolaco

Derivados do Ácido Fenilacético

Diclofenaco de Sódio, Aceclofenaco

Derivados do Ácido Propiônico

Ibuprofeno, Naproxeno, Flurbiprofeno, Cetoprofeno, Fenoprofeno,

Oxaprozino, Indoprofeno, Ácido Tiaprofênico

Derivado do Ácido Enólico (Oxicam)

Piroxicam, Meloxicam, Tenoxicam, Sudoxicam, Isoxicam, Ampiroxicam, Droxicam, Lornoxicam, Cinoxicam, Ampiroxicam, Pivoxicam

Derivado do Ácido Naftilacético

Nabumetona, Proquazona

Derivados do Ácido Carbâmico

Flupirtina

\section{Inibidores Seletivos Da Cox-2}

Derivado da Sulfonanilida

Nimesulida

Derivado do Ácido Indolacético

Etodolaco

Derivado Furanona Diarilsubstituído

Rofecoxib

Derivado Pirazol Diarilsubstituído

Celecoxib

Derivado Bipiridínico Diarilsubstituído

Etoricoxib

Derivado Isoxazol Diarilsubstituído

Valdecoxib

Fonte: CARVALHO, 201011. 


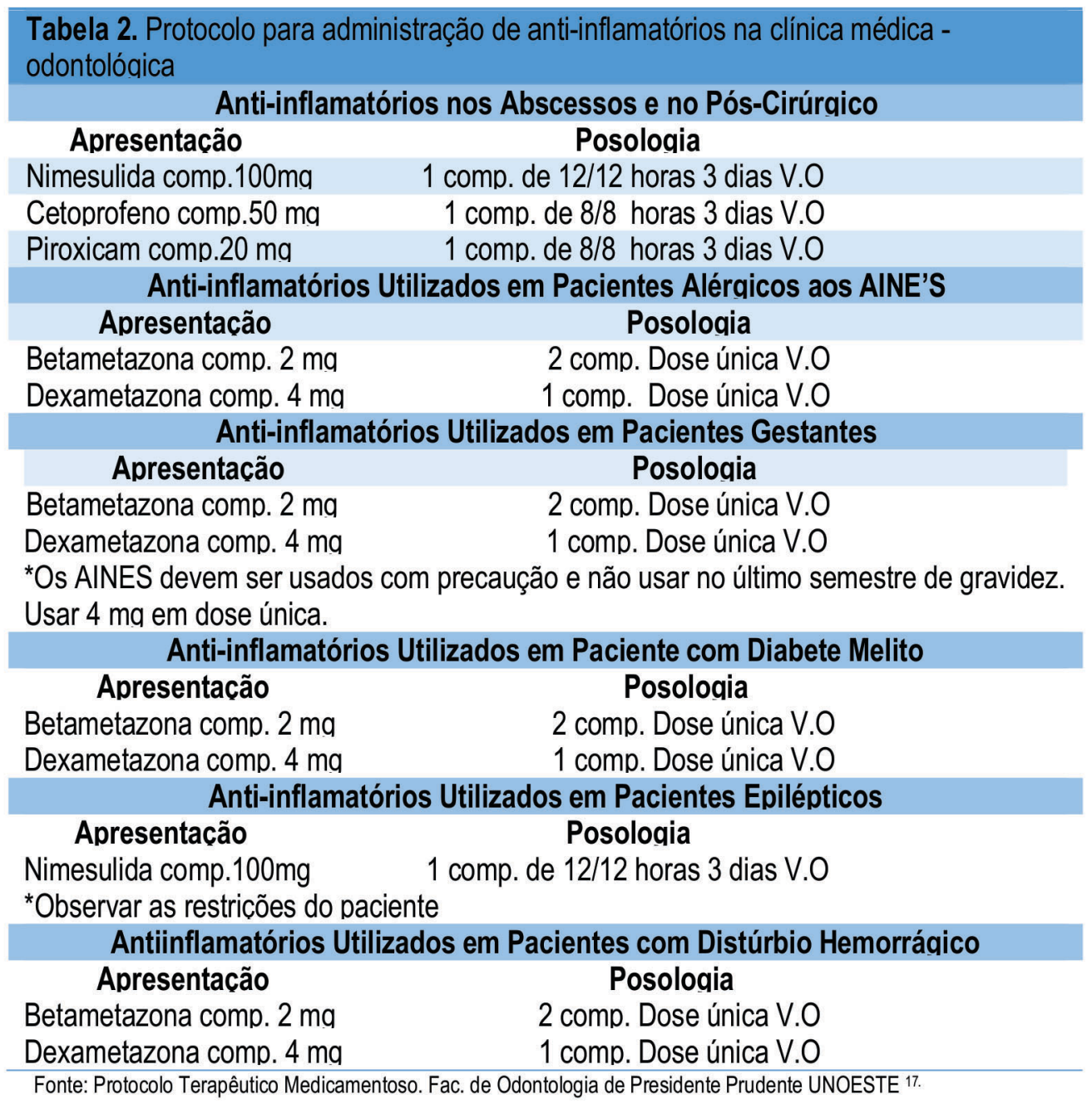

músculoesqueléticas, artrite reumatoide, lúpus eritematoso sistêmico, esclerose sistêmica progressiva, poliarterite nodosa, dismenorreia primária, mastocitose sistêmica, e também no traumatismo pós cirúrgico evitando dor e a instalação do edema. São também eficazes como adjuvantes no tratamento da gota aguda, osteoartrose, artroplastia e fibrose cística. Na infância e adolescência são indicados o AAS, naproxeno, ibuprofeno e tolmentina para controle da dor, febre e inflamação. Por outro lado a utilização desses fármacos é contraindicada em pacientes que apresentam histórico de hipersensibilidade alérgica e úlcera péptica, havendo restrições de uso para gestantes, idosos e recém-nascidos ${ }^{12,15}$.

Complicações associadas ao uso de medicamentos dessa classe chamam a atenção por apresentarem não apenas um risco aos usuários, mas vários, o que implicam problemas gastrintestinais, cardiovasculares, cerebrovasculares e trombóticos, renais, gestacionais e fetais que são atribuídos ao seu uso crônico e irracional, o que pode levar a sérias complicações $^{8,12}$. A escolha adequada de um AINE's no tratamento dependerá das características de cada paciente, fatores de riscos/benefícios e efetividade terapêutica desejada, podendo haver possibilidades de respostas diferenciadas.

Os conhecimentos prévios relacionados à medicação são atribuídos ao profissional, que tem o dever de transmitir e elucidar as informações necessárias a fim de obter o sucesso do fármaco empregado no tratamento. A prática errônea da utilização desses fármacos é possível que o profissional venha a desempenhar papel cruciante na possibilidade da ocorrência dos efeitos colaterais negativos produzidos pelo medicamento. Desta forma entre as muitas especialidades farmacêuticas é de bom alvitre optar pelos coxibes, devido à menor ocorrência dos efeitos irritativos e hemorrágicos no trato gastrointestinal que são comumente apresentados. 


\section{CONCLUSÃO}

Com base nesta revisão podemos aferir que nos esquemas posológicos adotados para os diferentes casos clínicos, tanto os coxibes quanto os AINE's não seletivos, têm diferentes graus de segurança cardiovascular, em particular o rofecoxibe e diclofenaco, os quais apresentam maior prevalência, associados à morbimortalidade cardiovasculares. Por isso, devem ser prescritos com cautela, inclusive a indivíduos sadios ${ }^{17}$. A enzima COX-2 resulta na formação de PG, em especial PGI2. Esta promove efeito antitrombótico, vasodilatação e redução da agregação plaquetária, essa enzima sendo inibida através do uso de inibidores seletivos da COX2 , podem afetar diretamente o equilíbrio entre alguns eicosanóides (TXA2 e PGI2), levando ao aumento de eventos trombóticos e cardiovasculares

Os conhecimentos técnicos relacionados ao uso da medicação são atribuídos ao profissional, que tem o dever de transmitir e elucidar as informações necessárias para o paciente, no objetivo de obter o sucesso farmacoterapêutico. A escolha adequada de um AINE's no tratamento dependerá das características individualizadas de cada paciente, fatores de riscos/ benefícios e efetividade terapêutica desejada, podendo assim haver possibilidades de respostas diferenciadas Ainda deverá basear-se na conveniência e necessidade da administração, o que contribuirá para o sucesso do tratamento na inibição do processo inflamatório.

\section{REFERÊNCIAS}

1. Buer JK. Origins and impact of the term 'NSAID', Inflammopharmacology, 2014; 22(5):263-7.

2. Bertolini A, Ferrari A, Ottani A, Guerzoni S, Tacchi R, Leone S. Paracetamol: new vistas of an old drug. CNS Drug Reviews. 2006; 12(3-ara):250-75.

3. Lafont $\mathrm{O}$. From the willow to aspirin. Revue d'histoire de la pharmacie. 2007; 55(354):209-16.

4. Brunton LL, John SL, Keith LP. Goodman \& Gilman's The Pharmacological Basis of Therapeutics $11^{\mathrm{a}}$ ed. Rio de Janeiro: McGraw-Hill, 2006.

5. Khanapure SP, Garvey DS, Janero DR, Letts LG. Eicosanoids in inflammation: biosynthesis, pharmacology, and therapeutic frontiers. Current Topic in Medicinal Chemistry. 2007; 7(3):311-40.

6. Süleyman H, Demircan B, Karagöz Y. Anti-inflammatory and side effects of cyclooxygenase inhibitors. Pharmacological reports. 2007; 59(3):247-58.

7. Bertolini A, Ottani A, Sandrini M. Dual acting antiinflammatory drugs: a reappraisal. Pharmacological Research. 2001; 44(6):437-50.

8. Loureiro APM. Nefrotoxicantes e Mecanismos de Lesão Renal (PDF).

9. Gotzche P. Deadly Medicines and Organised Crime: How Big Pharma Has Corrupted Healthcare [S.I.: s.n.].2013.

10. FitzGerald GA, Patrono $C$. The coxibs, selective inhibitors of cyclooxygenase-2. The New England Journal of Medicine.2001; 345(6): 433-442.

11. Carvalho WA. Antiinflamatórios Não Esteroides, Analgésicos, Antipiréticos e Drogas Utilizadas no Tratamento da Gota. In: Silva P. Farmacologia. 8. ed. Rio de Janeiro: Guanabara Koogan, 2010. Cap. 45, p. 439466.

12. Silva JM. Mendonça PP, Partata AK. Anti-Inflamatório NãoEsteroides e Suas Propriedades Gerais. Revista Científica do ITPAC, 2014; 7(4): 5.

13. Monteiro ECA, Trindade JMF, Duarte ALBP, Chahade WH. Os antiinflamatórios não esteroidais (AINEs). Revista Temas de Reumatologia Clínica. 2008; 9(2): 53-56.

14. Ferreira MBC, Wannmacher L. Anti-inflamatórios não esteróides. In: Fuchs FD, Wannmacher L, Ferreira MBC. Farmacologia Clínica - Fundamentos da Terapêutica Racional. 3 ed. Rio de Janeiro: Guanabara Koogan, 2006. Cap. 23, p. 296-304

15. Burke A, Fitzgerald GA. Analgésicos-antipiréticos; Farmacoterapia da gota. In: Hardman JG., et. al. Goodman \& Gilman - As Bases Farmacológicas da terapêutica. 11. ed. Rio de Janeiro: McGraw-Hill, 2006. Cap. 26, p. 601-637.

16. Hilário MOE, Terreri MT, Len CA. Antiinflamatórios nãohormonais: inibidores da ciclooxigenase 2. Jornal de Pediatria.Rio Janeiro, 2006; 82(5):206-212.

17. Parisi AGS, Amaral COF, Coelho COL, Oliveira DL, Pizi ECG, Souza FAD et al. Protocolo Terapêutico Medicamentoso. Faculdade de Odontologia de Presidente Prudente. UNOESTE.

18. Barreto RC, Pereira GAS. Farmacoterapia na Clínica Odontológica. $1^{\mathrm{a}}$ ed. João Pessoa: Editora Universitária. 2008. Cap. 9, 137-164.

Correspondência

Rosimar de Castro Barreto

Endereço: Rua João Navarro Filho -333 - Bessa

João Pessoa - Paraíba - Brasil - 58037-108

E-mail: rosimar@ccs.ufpb.br 